\title{
Monitoring Breathing Frequency, Pattern, and Effort
}

\author{
J Brady Scott and Ramandeep Kaur
}

\author{
Introduction \\ Clinical Significance of Breathing Frequency \\ Is Breathing Frequency Undervalued? \\ Is Accuracy a Problem? \\ Monitoring Breathing Frequency \\ Manual Counting of Breathing Frequency \\ Exhaled Carbon Dioxide \\ Acoustic Breathing Frequency Monitoring \\ Other Technology for Breathing Frequency Monitoring \\ Noninvasive Minute Ventilation Monitoring \\ Monitoring Breathing Effort \\ Work of Breathing \\ Monitoring Airway Occlusion Pressure \\ Monitoring Dyspnea \\ Conclusions
}

\begin{abstract}
Monitoring respiratory values such as breathing frequency, minute ventilation, breathing effort, and dyspnea are common in acute care. There is evidence that accurate monitoring and interpretation of these values leads to early identification and treatment of impending respiratory failure. Despite this evidence, some values, such as breathing frequency, are largely undervalued in the clinical setting. The undervaluation of breathing frequency is complex and will require a multifaceted approach, including education and improved technology, to reestablish its clinical potential. Many questions remain regarding how to most efficiently and effectively monitor other respiratory values, like noninvasive minute volume and breathing effort, as well. As technology continues to improve alongside the understanding of respiratory physiology, clinicians are able to apply basic clinical assessment skills and technology together to improve patient safety and outcomes. Key words: breathing frequency; vital signs; physical examination; patient assessment; end-tidal carbon dioxide; capnography; capnometry; dyspnea; minute ventilation; monitoring. [Respir Care 2020;65(6):793-806. (C) 2020 Daedalus Enterprises]
\end{abstract}

\section{Introduction}

Vital sign assessment is common in virtually all clinical areas. ${ }^{1-4}$ While important, vital signs are often overlooked,

The authors are affiliated with the Department of Cardiopulmonary Sciences, Division of Respiratory Care, Rush University, Chicago, Illinois.

Mr Scott presented a version of this paper at the 58th RespiRatory CARE Journal Conference, held June 10-11, 2019, in St. Petersburg, Florida.

Mr Scott has disclosed a relationship with Ventec Life Systems and Teleflex. Ms Kaur has disclosed no conflicts of interest. poorly documented, or undervalued clinically. ${ }^{3,4}$ Breathing frequency $(f)$, in particular, has been reported to be clinically underappreciated. This is concerning because $f$ is an early indicator of clinical deterioration and often precedes changes in other vital signs, like a decrease in

Correspondence: J Brady Scott MSc RRT RRT-ACCS AE-C FAARC, Department of Cardiopulmonary Sciences, Division of Respiratory Care, Rush University, Armour Academic Center, 600 S. Paulina, 751 AAC, Chicago, IL 60607. E-mail: jonathan_b_scott@rush.edu.

DOI: $10.4187 /$ respcare.07439 
blood pressure. ${ }^{4}$ Monitoring the respiratory status of a patient, however, extends beyond $f$ alone. Noninvasive minute ventilation monitoring can provide information about tidal volume and may be a better indicator of ventilatory status. Work of breathing and dyspnea monitoring allows clinicians to apply specific therapeutic strategies to overcome the increase of each.

The purpose of this paper is to bring further attention to the issue related to poor $f$ assessment and documentation. Additionally, methods to improve the monitoring of $f$ are examined. Other monitored parameters, like minute ventilation, work of breathing, and dyspnea, are also described.

\section{Clinical Significance of Breathing Frequency}

The normal $f$ in an adult patient is $12-20$ breaths/min and may vary normally with increased physical activity and sleep. ${ }^{1}$ In the context of illness, an increased $f$ (ie, tachypnea, defined as a $f>20$ breaths/min) may indicate conditions such as hypoxia, pain, cardiac insufficiency, or metabolic disturbances like lactic acidosis. ${ }^{1}$ A decreased $f$ (ie, bradypnea, defined as a $f<12$ breaths/min) may indicate neurologic depression or even more severe cases of hypoxia. ${ }^{1}$

There is considerable evidence that $f$ abnormalities can be early indicators of clinical deterioration. ${ }^{2-7}$ In 1993, Fieselmann et $\mathrm{al}^{5}$ conducted a retrospective case-controlled study that compared $72 \mathrm{~h}$ of vital signs in subjects who experienced cardiopulmonary arrest $(n=59)$ to $72 \mathrm{~h}$ of prearrest vital signs of subjects who did not experience cardiopulmonary arrest. They reported that $f$ was a significant $(P<.001)$ predictor of cardiopulmonary arrest, when controlled for age and gender. They also found that $f>27$ breaths/min was a predictor of cardiopulmonary arrest. Interestingly, heart rate and blood pressures were not predictors of cardiopulmonary arrest. ${ }^{5}$

In 2003, Subbe et al ${ }^{6}$ sought to evaluate a Modified Early Warning score that included systolic blood pressure, heart rate, $f$, temperature, and neurologic score. They noted that changes in $f$ were of a much greater magnitude than changes in heart rate and blood pressure in subjects at risk for clinical deterioration. Further, they noted their data suggests that $f$ is the best discriminator of identifying high-risk patients. ${ }^{6}$ Goldhill et $\mathrm{al}^{7}$ noted in their study a mortality rate associated with $f$ ranges: $21 \%$ of subjects with $f=25-29$ breaths/min died; $28 \%$ of subjects with $f=30-34$ breaths/min died; and $41 \%$ of subjects with $f \geq$ 35 breaths/min died. ${ }^{7}$

\section{Is Breathing Frequency Undervalued?}

In 2008, Cretikos et $\mathrm{al}^{3}$ placed considerable emphasis on the idea that $f$ is a largely neglected vital sign. They pointed out that evidence up to that time suggested that $f>20$ breaths/min in adult patients would indicate illness, and that $f>24$ breaths/min would indicate critical illness. ${ }^{3}$ Despite this, emphasis was not being placed on $f$ monitoring in medical textbooks and other clinical training. They summarized that $f$ is a poorly recorded vital sign and that elevated $f$ is a strong and specific indicator of events such as cardiac arrest. They also added that pulse oximetry is not a replacement for $f$ measurement, that staff should be educated on the importance of $f$, and that hospitals should encourage appropriate responses to changes in $f^{3}$.

An audit of medical notes was conducted in 2010 of 594 adult patients who presented to an emergency department. ${ }^{2}$ The notes were picked randomly, and the study author recorded whether $f$ was documented by clinicians, as well as the subjects condition during the subjects emergency department visit. It was noted that $f$ was only documented $29 \%$ of the time. With that said, in most subjects with respiratory problems, $f$ was documented. Specifically, $91 \%$ of subjects who presented with shortness of breath had their $f$ documented. Other conditions had lower percentages of $f$ documentation. For example, chest pain and abdominal pain were lower at $63 \%(n=53)$ and $31 \%(n=74)$, respectively. ${ }^{2}$ The author correctly notes concern for this variation in $f$ documentation, because conditions that cause abdominal pain may result in $f$ changes. $^{2}$

In 2014, Ansell et $\mathrm{al}^{8}$ reported the results of a qualitative study that investigated nursing practices with regard to monitoring and documenting $f$. They conducted semi-structured telephone interviews on 10 ward nurses from 3 different hospitals in New Zealand. Although limited to only 10 nurses, the study offered interesting insight on why $f$ may be unmonitored or undocumented. Results cited time pressure, work interruptions, and clinical judgment as reasons that $f$ was not assessed or documented. ${ }^{8}$ It is noted that $f$ remains the only vital sign largely assessed manually, contributing to time pressure and consistency issues. Troublingly, there is also a suggestion that $f$ is not valued as a primary assessment tool when compared to others. The authors conclude that this may be a result of a lack of understanding of respiratory physiology. ${ }^{8}$ These results have to be interpreted cautiously due to the small scale and scope, but they do provide insight on the realities that nurses and other clinicians face each day at the bedside in terms of time pressure and other clinical responsibilities.

Flenady et $\mathrm{al}^{9}$ sought to explain why emergency department nurses often fail to assess or document $f$ assessments. They noted that despite an abnormal $f$ being a good indicator of clinical deterioration, $f$ is a poorly documented vital sign. ${ }^{9,10}$ Using classic grounded-theory methodology, they analyzed qualitative data gathered from 79 emergency department nurses in Australia. They found, like other studies, that $f$ assessments were either absent or erroneously documented in the emergency department setting. They also reported that, despite significant evidence that 
abnormal $f$ is indicative of clinical deterioration, emergency department nurses can justify reasons for it not to be properly assessed or documented. They noted that nurses are aware of the best practices for $f$ assessment; however, they feel it is an unnecessary action for many of their patients. The behavior is explained by the authors as strategies that emergency department nurses may employ, such as compensating, minimalizing, and trivializing. ${ }^{9}$ According to the authors, a compensating behavior suggests that time spent with the patient could be spent more wisely on tasks other than assessing $f$. So, according to their theory, the transgression in behavior is rationalized by the patient benefiting from other tasks, instead of the one that is demanded by the organization. Minimalizing behavior is simply explained by nurses essentially not believing that patient outcomes would be different if $f$ were accurately assessed and recorded. Finally, trivializing could be explained by the need to cut corners when time with the patient is limited. Additionally, the act of assessing may be considered trivial in an emergency department setting due to the high acuity of other patients and even emergency department social norms. ${ }^{9}$ Flenady et $\mathrm{al}^{9}$ recommended that organizations assess how education is delivered to their staff. They noted that education should be delivered in a way that considers diverse human factors and the culture of the workplace. Considering these factors may positively influence change in practice and adherence to evidence-based practices. ${ }^{9}$

It appears that $f$ is generally undervalued as a vital sign. It is not clear why this may be true, but the lack of understanding of respiratory physiology, time constraints, work load, and other reasons that are not yet clearly understood play a role.

\section{Is Accuracy a Problem?}

As Flenady et $\mathrm{al}^{9}$ reported, $f$ is not only omitted for various reasons, but it also is recorded inaccurately in many cases. Others have noted inaccuracies in $f$ measurements as well. In 2005 , Lovett et al ${ }^{11}$ noted that $f$ often was recorded inaccurately in the emergency department triage setting. In their study, neither triage nurses nor transthoracic impedance plethysmography devices were as accurate as $f$ measurements made using a standard 60-s observation. ${ }^{11}$ Mukkamala et al ${ }^{12}$ counted $f$ in their hospital for $60 \mathrm{~s}$ and compared the findings to the nursing values reported in the patient chart. It was noted that the nurses had reported $f=$ 20 about $50 \%$ of the time (234 of 467) compared to $3 \%$ (13 of 469) by the study team. The distribution of recordings in their study overall did not show a statistical difference $(P=$ $.105)$; however, the authors noted a potential for clinical importance. They cited that incorrect recording of $f$ could delay early recognition of conditions such as sepsis. ${ }^{12}$
In 2013, Semler et $\mathrm{al}^{13}$ sought to evaluate the accuracy of recorded $f$ in hospitalized, non-ICU patients. In their single-day, resident-led, prospective observational study of recorded and directly observed vital signs from 6 different academic centers, vital signs were collected from 368 subjects. The median $f$ was 16 breaths/min (interquartile range 14-20 breaths/min) for directly observed measurements and 18 breaths/min for recorded values (interquartile range 18-20 breaths/min). The median difference of 2 breaths/min was statistically significant $(P<.001)$. Interestingly, $f$ of 18 or 20 breaths/min accounted for the majority of the documented values (71.8\%), but these values were actually observed only $13 \%$ of the time. The authors concluded that documented $f$ in hospitals may actually be higher than directly observed and are likely to be recorded as 1820 breaths/min.

Philip et $\mathrm{al}^{14}$ evaluated $f$ assessments made by physicians using 2 different assessment techniques. In their study, physicians were asked to view videos that depicted different $f$ values and to do a spot assessment (ie, estimate $f$ by looking at a patient for no longer than $12 \mathrm{~s}$ ) or formal assessment (ie, counting $f$ for $30 \mathrm{~s}$ and then multiplying by 2 , or counting for a full minute). Using the spot assessment, only $48 \%$ of clinicians identified $f$ correctly. Using the formal assessment, $81 \%$ of clinicians identified $f$ correctly. Clinicians were able to identify tachypnea $(f=72$ breaths/min) more accurately, using either method, than bradypnea $(f=6$ breaths/min). The authors noted that both methods had a high level of inaccuracy, but formal assessment was superior. Of the 54 participants, 52\% stated that they use spot assessments in their clinical practice. Regarding accuracy of $f$ documentation in the medical note, $0 \%$ of them reported that $f$ was accurate all of the time. Only $20 \%$ reported they felt it was accurate most of the time. The majority (72\%) reported that they believed $f$ was accurate in the chart sometimes. A small percentage (7\%) felt it was never accurate. ${ }^{14}$

The significance of $f$ inaccuracy cannot be overstated, nor can it be ignored, because it is possible that tachypnea or bradypnea are not being clinically observed and documented properly. This could delay timely response to serious conditions that could lead to preventable death. Despite various explanations regarding the cause of this problem, a meaningful solution remains elusive.

\section{Monitoring Breathing Frequency}

\section{Manual Counting of Breathing Frequency}

There are 2 main ways to monitor $f$ : manually, where $f$ is counted by a clinician, and continuously, where $f$ is measured by a device. It appears that when manual $f$ is done, it should be counted for a full minute. ${ }^{15,16}$ Ideally, the patient would be unaware that the $f$ assessment is being made so as 
to avoid a conscious change in $f$, breathing pattern, and depth. ${ }^{15,16}$ The time-consuming nature of $f$ assessment (ie, counting for a whole minute) has been cited as a reason that clinicians have sought alternative methods to perform the task. ${ }^{8,17-19}$ As such, Takayama et al ${ }^{19}$ evaluated 2 quickcheck methods and compared them to the standard accepted method of counting $f$ for a full minute (Fig. 1). One of the quick-check methods was to simply count $f$ for $15 \mathrm{~s}$ and multiply the count by 4 (ie, the 15 -s quadruple method). The other method is a novel method of counting $f$ by counting the time needed for a single breath and dividing 60 by the time for a single breath (ie, the breathing time measurement). The investigators reported that, when compared to the standard 1-min count, the 15-s quadruple method consistently overestimated $f$. The breathing time measurement method had a better agreement with the standard 1-min count. Despite this interesting finding and the potential for further study and use in the clinical setting, the authors noted that this method may not be a good alternative to the 1-min method in the presence of an abnormal breathing pattern. ${ }^{19}$ Future research aimed at finding reliable quickcheck methods is certainly warranted. Until more is known about quick-check methods, clinicians will need to continue to utilize the $1-\min f$ assessment and technology to provide a more accurate assessment of $f$.

\section{Exhaled Carbon Dioxide}

Technology has enhanced clinicians' ability to continuously monitor $f$ and breathing pattern. Exhaled carbon dioxide $\left(\mathrm{CO}_{2}\right)$ technology (ie, capnography/capnometry) is commonly used and has clinical value in endotracheal tube confirmation, detection of airway dislodgement, and monitoring of respiratory depression. ${ }^{20-22}$ In fact, this technology has been recognized in practice guidelines and standards by such organizations as the American Society of Anesthesia, American Association for Respiratory Care, the Joint Commission, and American Heart Association. ${ }^{20}$ Exhaled $\mathrm{CO}_{2}$ can be measured in a number of ways, including through a breathing circuit or noninvasively with a nasal cannula device that is designed to capture exhaled $\mathrm{CO}_{2}$ (Fig. 2). Exhaled $\mathrm{CO}_{2}$ can be displayed numerically (as $\mathrm{P}_{\mathrm{ETCO}_{2}}$, partial pressure of end-tidal $\mathrm{CO}_{2}$ ) and graphically as a $\mathrm{CO}_{2}$ waveform called a capnogram (Fig. 3). A numerical $f$ is displayed, as well.

Exhaled $\mathrm{CO}_{2}$ can provide clinicians a reliable way to monitor $f$ and may provide valuable information pertaining to respiratory dysfunction or depression. ${ }^{22}$ Perhaps the main value of exhaled $\mathrm{CO}_{2}$ monitoring is that it provides a more complete picture of respiratory status when compared to pulse oximetry alone. Pulse oximetry provides important information about the oxygenation status of a patient, but it does not provide information that pertains to ventilatory function. ${ }^{20}$ Continuous exhaled $\mathrm{CO}_{2}$
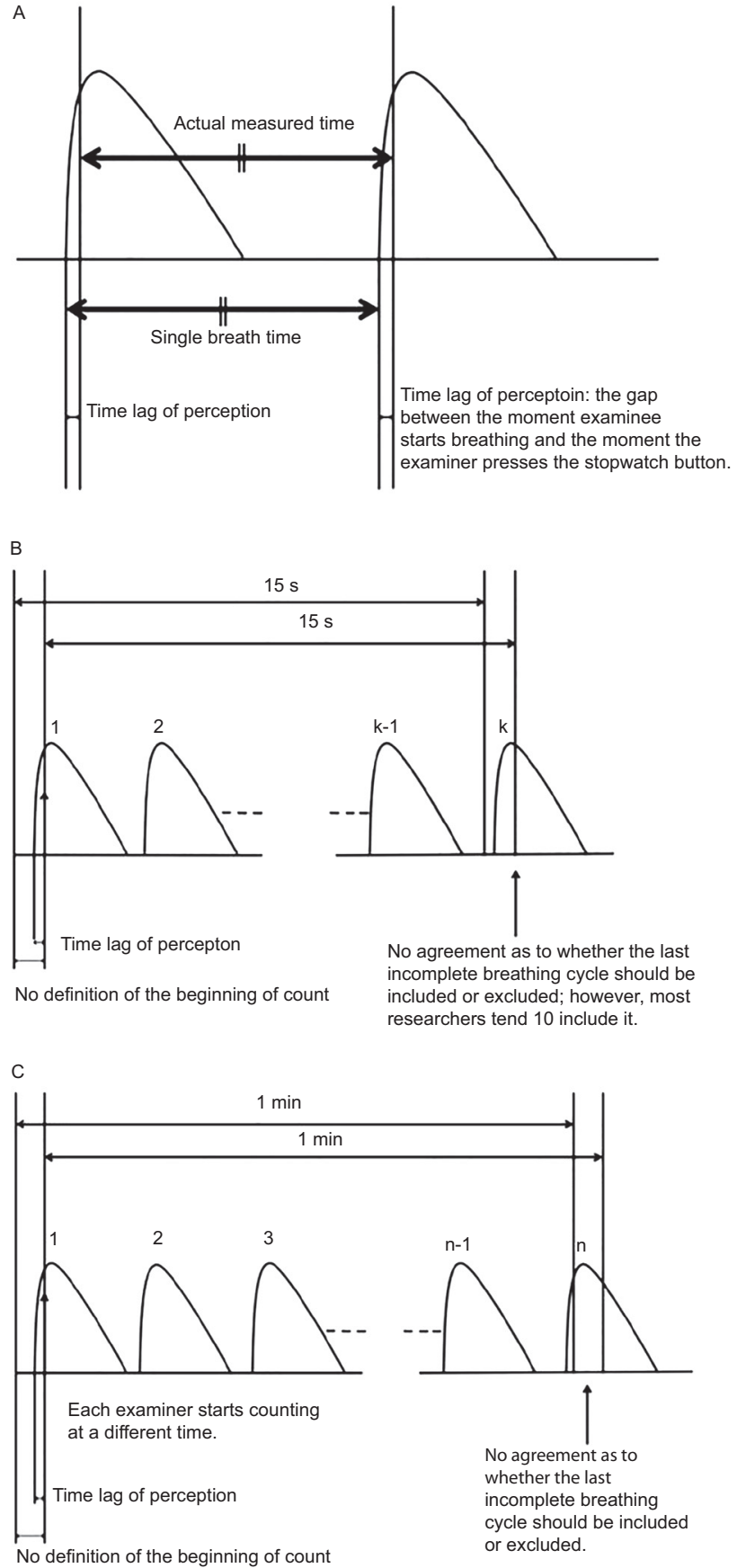

Fig. 1. Graphic representation of 3 methods for counting breathing frequency. (A) Breathing time measurement. (B) 15-s quadruple method. (C) Count for a full minute. From Reference 19.

monitoring is more effective for the early detection of ventilatory compromise compared to both visual assessment and pulse oximetry. ${ }^{21,23,24}$

Although exhaled $\mathrm{CO}_{2}$ monitoring is generally recommended as a way to monitor adequacy of ventilation, especially in the setting of patient-controlled analgesia, ${ }^{20,21,23,24}$ 


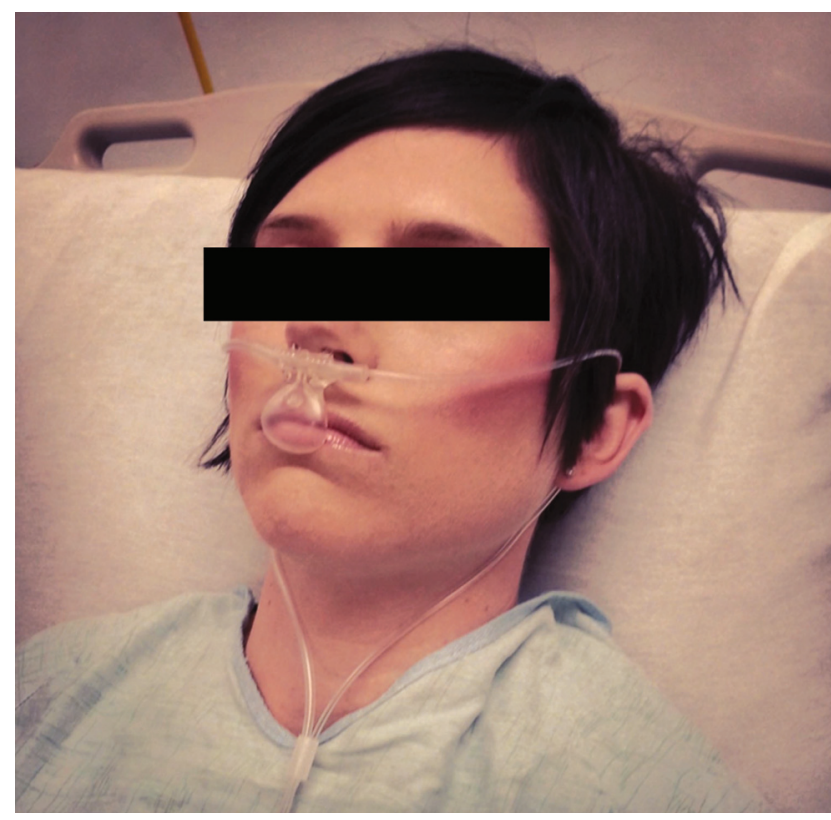

Fig. 2. Example of a cannula designed specifically to capture exhaled $\mathrm{CO}_{2}$ from the mouth and nares.

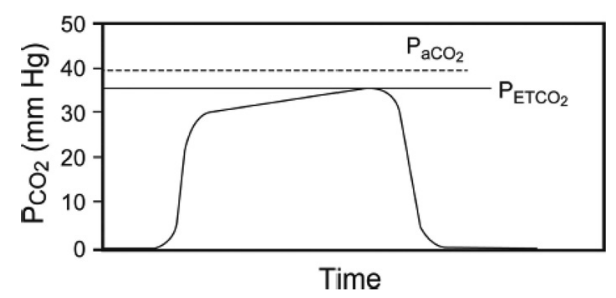

Fig. 3. Capnogram showing difference in $\mathrm{P}_{\mathrm{aCO}}$ and partial pressure of end-tidal $\mathrm{CO}_{2}\left(\mathrm{P}_{\mathrm{ETCO}}\right)$. From Reference 20.

some questions remain. ${ }^{25-27}$ A recent review of capnography use in the emergency department setting during procedural sedation and analgesia revealed it did not result in significance decreases in clinically important events, such as hypoxia or need for airway interventions..$^{25,26}$ This requires further study, especially in high-risk patients, because capnography fills a diagnostic void left by pulse oximetry and respiratory assessment alone. ${ }^{25,28}$ Although exhaled $\mathrm{CO}_{2}$ is relatively easy to use for clinicians, some issues with patient adherence exist. In a study to promote the use of exhaled $\mathrm{CO}_{2}$ monitoring of patients at risk for opioid-induced respiratory depression, Carlisle ${ }^{21}$ noted that most nurses (92\%) agreed or strongly agreed that alarms wake patients up while sleeping. Additionally, $65 \%$ of nurses agreed or strongly agreed that patients often refuse to wear the cannula device. ${ }^{21}$

Monitoring exhaled $\mathrm{CO}_{2}$ is tremendously valuable in detecting variations in $f$ and breathing pattern that may be indicative of respiratory dysfunction. ${ }^{28}$ It is widely recommended for the evaluation and assessment of ventilation, and it enhances patient safety. Generally, capnography/capnometry devices are easy to use in the clinical setting. ${ }^{21}$ With that said, many issues remain, such as patient selection, patient adherence, and clinician training. Clinicians must be trained to understand the technology, normal and abnormal values, waveforms, and trend information. ${ }^{28}$ The cannulas used to detect exhaled $\mathrm{CO}_{2}$ can be irritating to patients, especially if they do not understand the value in terms of monitoring and safety. ${ }^{21}$ Thus, patient adherence is a very real concern. Patients can easily remove or displace the device if they deem it uncomfortable. Other devices designed to monitor $f$ have been introduced as an alternative to exhaled $\mathrm{CO}_{2}$ monitoring.

\section{Acoustic Breathing Frequency Monitoring}

Acoustic $f$ monitors have been introduced as a way to continuously monitor $f$ in various clinical settings. These devices utilize a sensor placed on the neck to detect air flow in the pharynx produced during inhalation and exhalation. ${ }^{29}$ In general, these devices are quite effective compared to exhaled $\mathrm{CO}_{2}$ monitoring. ${ }^{30-33}$ In the first study comparing the newer acoustic technology to capnometry, Mimoz et $\mathrm{al}^{30}$ reported that it correlated well with capnometry in the evaluation of $f$ in extubated postsurgical subjects. Ramsay et $\mathrm{al}^{33}$ also reported acoustic monitoring to be effective in postsurgical subjects. In their study, the acoustic monitor was more accurate and precise in detecting $f$ when compared to capnometry; however, the findings were modest and the clinical importance is unclear. ${ }^{33}$

More recent studies have also demonstrated the potential usefulness of acoustic $f$ monitoring. In subjects under intravenous anesthesia for dental procedures, Ouchi et $\mathrm{al}^{34}$ noted that acoustic $f$ was useful when compared to capnography. However, they note that this technology may be limited when a dental air turbine is used. Compared to electrocardiographically derived $f$, acoustic monitoring may offer more clinically useful information in unstable trauma patients. ${ }^{35}$ In 2019, Ishikawa et al ${ }^{36}$ reported in a case report that an abrupt change in $f$ (eg, from 8 to 30 breaths/min) was detected in a patient who had recently undergone a thyroidectomy. The patient was subsequently intubated and surgically treated for a hematoma due to postoperative bleeding. The authors noted that acoustic $f$ monitoring allowed the early detection of respiratory complications postoperatively. ${ }^{36}$

In contrast to the overall positive studies supporting the use of acoustic $f$ monitoring, McGrath et $\mathrm{al}^{37}$ noted that, while acoustic $f$ monitoring may augment a pulse oximetrybased surveillance system in postsurgical patients, the technology may not add significant detection of clinical deterioration. They also reported that adherence to the device was only $57 \%$, noting a $22.7 \%$ refusal rate. ${ }^{37}$ 
Other Technology for Breathing Frequency Monitoring

Pulse oximetry-derived $f$ monitoring, or a photoplethysmographic respiratory monitor, detects changes in intrapleural pressures that are transmitted to the cardiovascular system. ${ }^{29}$ Eisenberg et $\mathrm{al}^{38}$ evaluated this technology in healthy subjects and compared it to acoustic $f$ monitoring; both methods were compared to capnography as well. The researchers reported that the pulse oximetry-derived $f$ was more likely to detect bradypnea and may be more reliable than acoustic $f$ monitoring in the presence of routine patient activities. Although promising, more studies are needed because this technology has not yet shown clinical superiority over capnography or acoustic $f$ monitoring. ${ }^{29}$

Thoracic impedance devices have also been evaluated and compared to acoustic $f$ monitoring and capnography. Guechi et $\mathrm{al}^{39}$ noted that acoustic $f$ monitoring was more accurate than thoracic impedance in subjects hospitalized for drug or alcohol poisoning. Similarly, Frasca et $\mathrm{al}^{40}$ determined that acoustic $f$ monitoring was more precise than thoracic impedance for $f$ measurement in obese subjects. In 2018, a new noninvasive, wireless, body-worn device that is able to continuously monitor $f$ was compared to capnography on subjects admitted to an acute medical unit. ${ }^{41}$ The study demonstrated that this new technology measures $f$ comparatively to capnography. ${ }^{41}$ The impact of this kind of technology is unknown, but could, like acoustic $f$ monitoring, offer an alternative to exhaled $\mathrm{CO}_{2}$ monitoring in patients who are intolerant of the nasal interface. Further clinical investigation is warranted.

\section{Noninvasive Minute Ventilation Monitoring}

Minute ventilation refers to movement of air into and out of the lungs within $1 \mathrm{~min}$ and is a product of $f$ and tidal volume $\left(\mathrm{V}_{\mathrm{T}}\right) . \mathrm{V}_{\mathrm{T}}$ is composed of dead space volume and alveolar volume. ${ }^{42}$ Dead space volume does not take part in gas exchange, whereas alveolar volume does. The volume of anatomic dead spaces remains the same in normal patients, but physiologic dead space varies based on disease process. Increasing $\mathrm{V}_{\mathrm{T}}$ /alveolar volume has a greater effect on gas exchange as compared to increasing $f$. Thus, the addition of $\mathrm{V}_{\mathrm{T}}$ monitoring can provide valuable insight on the ventilatory status of patients. Like $f$, minute ventilation has been shown to be an early indicator of respiratory failure. ${ }^{43}$

Monitoring minute ventilation noninvasively is clinically complicated because it requires special devices like spirometers or pneumotachometers to measure $\mathrm{V}_{\mathrm{T}}$. In patients who are not mechanically ventilated, clinicians have to apply masks or mouthpieces to the patient's face to capture the exhaled $\mathrm{V}_{\mathrm{T}}$. Further complicating this procedure, patients have voluntary control of their breathing, which makes these noninvasive techniques relatively unreliable. ${ }^{44}$

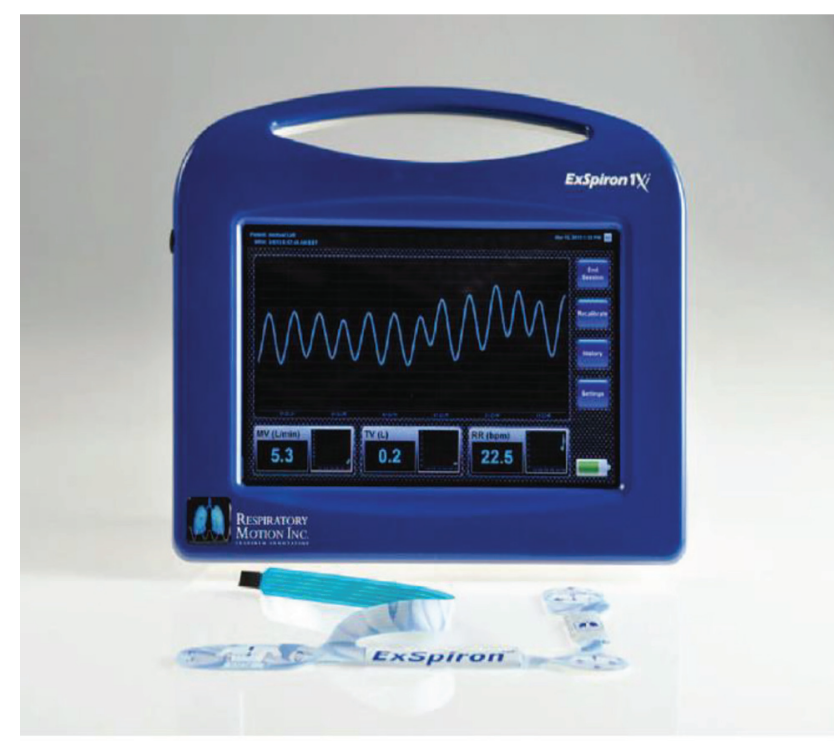

Fig. 4. Example of a respiratory volume monitor. From Reference 48.

These technical challenges and the cumbersome nature of obtaining $\mathrm{V}_{\mathrm{T}}$ have limited the widespread use of minute ventilation in spontaneously breathing patients. ${ }^{45}$ Instead, clinicians often rely on $f$ to monitor respiratory status in patients. Holley et al ${ }^{46}$ concluded, however, that $f$ measurement alone is insufficient to detect hypoventilation in subjects undergoing endoscopic procedures. In a prospective, observational study, $f$ was noted to be a poor predictor for respiratory depression, which was defined by low minute ventilation (ie, $<40 \%$ predicted minute ventilation for at least $2 \mathrm{~min}){ }^{47}$ Because the importance of minute ventilation monitoring has been recognized, efforts have been made to make it more clinically available.

Respiratory minute volume monitors (RVMs) (Fig. 4) are FDA-approved, noninvasive devices that continuously measure $\mathrm{V}_{\mathrm{T}}, f$, and minute ventilation via impedance-based thoracic electrodes in spontaneously breathing patients. ${ }^{48}$ The clinical utility and validity of RVMs has been explored in different patient populations and in different clinical areas. Voscopoulos et $\mathrm{al}^{49}$ evaluated the performance of RVMs in a broad cohort of ambulatory subjects, reporting that RVM measurement of $f$ has a relative error of $1.8 \%$ and measurement of minute ventilation and $\mathrm{V}_{\mathrm{T}}$ have a relative error of $<10 \%$ in spontaneously breathing subjects. In this study, the investigators observed no significant differences in $f, \mathrm{~V}_{\mathrm{T}}$, or minute ventilation measurements obtained with the RVM and a spirometer.

In a series of clinical cases, Schlesinger ${ }^{48}$ described the practical application of RVM in austere settings and in high-tech settings to help clinical decision making and to improve patient safety in critical care units. In subjects undergoing elective surgery with general anesthesia, Voscopoulos et $\mathrm{al}^{50}$ reported a close correlation (>94\%) 
between RVM-measured minute ventilation, $\mathrm{V}_{\mathrm{T}}, f$, and ventilator measurements collected from intubated subjects. Nichols et al $^{51}$ conducted a randomized controlled trial to examine the effectiveness of RVM in improving patient safety for those undergoing procedural sedation. In this trial, 65 subjects were randomly assigned to either a control group or a RVM intervention group. Anesthesiologists in the RVM intervention group were encouraged to use RVM values to titrate intravenous anesthetics to maintain minute ventilation within $40-80 \%$ of baseline value. The investigators reported a higher incidence of hypoventilation and apnea in the control group compared to the RVM intervention group. They concluded that RVM-based interventions have the potential to identify early signs of respiratory depression and may improve patient safety. In an observational study by Mehta et al, ${ }^{52}$ RVM was utilized to measure minute ventilation in obese and nonobese subjects undergoing procedural sedation. The study results indicated higher minute ventilation among obese subjects compared to nonobese subjects before, during, and after the surgery. The authors note that RVM could be useful in capturing real-time changes in obese patients' metabolic demands to provide more individualized care to these patients.

Utilization of RVM after extubation in postoperative patients has also been evaluated. Ianchulev et al ${ }^{53}$ noted that RVM was able to provide clinicians with comprehensive, quantitative information related to breathing pattern and trends in ventilation that are not available with other technology at the time of their study. ${ }^{53}$ The authors concluded that RVM shows real promise in improving patient safety in postextubation patients, among others. Other tools with the ability to monitor postextubation respiratory status, like the integrated pulmonary index, ${ }^{54}$ have been evaluated since the study by Ianchulev et al, ${ }^{53}$ but to our knowledge they have not been compared directly.

RVM provides a novel and noninvasive method to adequately and continuously monitor a wide variety of patient groups that are at risk of respiratory failure. Continuous respiratory monitoring with RVM could lead to early detection of clinical deterioration, and therefore it may allow for timely intervention. Although promising, there is a need for large-scale clinical studies to prove the clinical effectiveness and accuracy of RVM in preventing respiratory failure and improving patient outcome and safety.

\section{Monitoring Breathing Effort}

de Vries et $\mathrm{al}^{55}$ defined breathing effort as an energyconsuming activity of the respiratory muscles aimed at driving respiration. The effort of breathing is controlled by respiratory centers located in the brainstem to maintain adequate oxygenation and ventilation within the body. Any imbalance between respiratory effort, muscle capacity, and ventilatory demand predispose an individual to respiratory insufficiency. ${ }^{56}$ Increased breathing effort leads to muscle fatigue, abnormal breathing patterns, and respiratory distress. On the other hand, decreased breathing effort can cause muscular deconditioning and abnormal gas exchange. ${ }^{57}$ The measurement of breathing effort allows clinicians to assess respiratory mechanics in both intubated and nonintubated patients. This knowledge helps guide the application of appropriate medical treatment and therapies to improve respiratory function in patients with respiratory related illnesses.

The most common and traditional way to assess a patient's breathing effort is through physical examination. Tachypnea, accessory muscle use, nasal flaring, tracheal tug, and diaphoresis are the primary signs present in individuals with inefficient respiratory effort. ${ }^{58}$ In a study by Tulaimat et al, ${ }^{59}$ the authors sought to determine the agreement among physicians on the presence of physical signs of increased respiratory effort. They reported only a fair to moderate agreement among physicians when assessing signs of increased breathing effort. The authors recommended that clinicians use signs of respiratory distress like hypoxia and tachypnea in conjunction with respiratory effort to describe a patient's overall respiratory status. They also noted a further need to refine the method of physical examination when assessing increased breathing effort. ${ }^{59}$

Breathing effort can be measured directly or indirectly through various quantitative methods and techniques. Direct measurements of respiratory effort are work of breathing (WOB) and pressure-time product (PTP). Indirect measurements that act as a surrogate for breathing effort are airway occlusion pressure at 100 ms and diaphragmatic electric activity. ${ }^{60}$ There is a paucity of literature available on measuring breathing effort in spontaneously breathing subjects. The majority of the research published on assessing breathing effort appears to be confined to subjects receiving mechanical ventilation.

\section{Work of Breathing}

WOB reflects the energy needed to overcome the elastic load imposed by the lung and chest wall and the resistive loads imposed by the airways. The most widely used method to measure WOB is esophageal pressure measurements, which involves a catheter and balloon inserted into a patient's esophagus. Esophageal pressure obtained from the balloon is used as a surrogate for the inspiratory muscle pressure generated during contraction. ${ }^{61}$ Because WOB does not account for the isometric phase of inspiration, due to absent volume change, PTP was developed to accurately reflect respiratory efficiency by measuring 
energy expenditure during the dynamic and isometric phases of the respiratory cycle. ${ }^{62}$ PTP is the product of the time spent in muscle contraction during inspiration, as a percent of the total respiratory cycle, and pressure generated by inspiratory muscles during contraction.

Abnormalities in either lung or chest wall compliance or airway resistance can affect PTP and WOB. Posture, artificial airways, and noninvasive ventilation also can influence these measures. ${ }^{63}$ In a prospective crossover study by Deye et al ${ }^{64}$ inspiratory WOB and PTP were used to determine the influence of posture on breathing effort in difficult-towean subjects. The study reported that a semi-seated position at $45^{\circ}$ was significantly associated with lower WOB, PTP, and inspiratory effort. In another study to assess pulmonary mechanics before and after tracheostomy in subjects requiring prolonged mechanical ventilation, there was no significant difference in WOB and PTP before and after tracheostomy ${ }^{65}$ Based on these findings, Lin et a ${ }^{65}$ recommended that clinicians should be cautious when utilizing only WOB and PTP values to predict weaning outcome. Thus far, WOB and PTP measurements are typically used for research purposes. Due to its technical difficulties, invasive nature, and limitations, it has yet to be translated to the bedside for widespread clinical use.

Artificial neural networks are computer models that have the capability to perform higher-level functions. ${ }^{66}$ Artificial neural networks have been utilized in critical care settings to measure patient's WOB $\left(\mathrm{WOB}_{\mathrm{N}}\right)$ without the use of an esophageal balloon. This computerized technique gathers data from a respiratory monitor connected to pressure and flow sensor placed between the endotracheal tube and the Y-piece of ventilator circuit. In a prospective study by Banner et $\mathrm{al}^{67}$ to compare the relationship between invasively and noninvasively measured WOB per minute in intubated subjects, the authors reported a high correlation in measured WOB. In another study, Banner et al ${ }^{68}$ evaluated the role of noninvasively obtained $\mathrm{WOB}_{\mathrm{N}}$ per minute in determining extubation outcome. The results indicated that, compared to common weaning indices like $f, \mathrm{~V}_{\mathrm{T}}, f / \mathrm{V}_{\mathrm{T}}$, minute ventilation, oxygen saturation, and the ratio of physiologic dead space volume to $\mathrm{V}_{\mathrm{T}}$, $\mathrm{WOB}_{\mathrm{N}}$ per minute had the highest predictive accuracy, sensitivity, and positive and negative predictive value in predicting extubation outcome. This technique of measuring WOB without the use of an esophageal balloon is attractive and could find use in routine clinical practice if proven effective, valid, and reliable in larger clinical studies.

Vivier et $\mathrm{al}^{69}$ described another noninvasive method to estimate WOB using diaphragm ultrasonography. In this preliminary study, investigators enrolled 12 subjects requiring planned noninvasive ventilation after extubation. These subjects were studied during spontaneous breathing and during noninvasive ventilation at 3 different pressure support levels $\left(5,10\right.$, and $\left.15 \mathrm{~cm} \mathrm{H}_{2} \mathrm{O}\right)$. The diaphragm thickening fraction was calculated as ([thickness at inspiration - thickness at expiration]/thickness at expiration). It has been noted that overassistance or inadequate ventilatory support alters diaphragmatic function and leads to a loss of diaphragm thickness and atrophy. The study results indicated that higher levels of pressure support were associated with decreased PTP and diaphragm thickening fraction. In addition, thickening fraction was significantly correlated with PTP. ${ }^{69}$ Diaphragm ultrasonography provides a quantitative method to assess respiratory muscle effort in nonintubated patients, but further studies are needed to explore this technique as a way to monitor WOB.

\section{Monitoring Airway Occlusion Pressure}

Airway occlusion pressure was first described by Whitelaw et $\mathrm{al}^{70}$ as a way to indirectly measure the output of respiratory centers. ${ }^{71}$ It can be recorded either by occluding the entire respiratory cycle in unconscious humans or by occluding a brief period of inspiration in conscious patients. In conscious patients, only the first $100-150 \mathrm{~ms}$ of inspiration are measured. The airway pressures recorded during first $100 \mathrm{~ms}$ of inspiration are known as $\mathrm{P}_{0.1}$, this is a commonly used parameter to measure ventilatory drive. According to Whitelaw et al, ${ }^{72}$ normal occlusion pressure signifies an integrated central nervous system, spinal cord, peripheral nerve, and muscle fiber function.

$\mathrm{P}_{0.1}$ measurement requires a patient to inhale through an occluded airway, which causes a negative pressure generation that is captured via a pressure transducer. $\mathrm{P}_{0.1}$ is not dependent on the air flow, so it is unaffected by the mechanical properties of lung and chest wall, such as compliance and resistance. As a result, to assess respiratory drive, $\mathrm{P}_{0.1}$ outperforms other common parameters like $\mathrm{V}_{\mathrm{T}}$ and $f$, and it can be easily measured in both intubated and nonintubated patients. ${ }^{73}$ Most modern ventilators are equipped with the capability to automatically measure $\mathrm{P}_{0.1}$ in intubated patients, although the accuracy of absolute values of $\mathrm{P}_{0.1}$ depends on the ventilator design. ${ }^{74}$

Inefficient ventilatory drive, especially during mechanical ventilation, could lead to iatrogenic muscle damage and thus could prolong the recovery phase and duration of mechanical ventilation. ${ }^{75} \mathrm{P}_{0.1}$ measurements provide a quantitative way to detect insufficient or excessive breathing effort. In a prospective, interventional study, Alberti and colleagues $^{76}$ evaluated the role of breathing pattern, $\mathrm{P}_{0.1}$, and WOB in determining the appropriate level of pressure support in subjects with acute respiratory failure requiring ventilatory assistance. They found that that $\mathrm{WOB}$ and $\mathrm{P}_{0.1}$ progressively increased with a decrease in pressure support level. They also noted a significant correlation between WOB and $\mathrm{P}_{0.1}(\mathrm{r}=0.87)$. The correlation between WOB and $f$ was less significant $(\mathrm{r}=0.53)$ at decreased pressure 


\begin{tabular}{|c|c|c|c|c|}
\hline \multirow[t]{2}{*}{ Variable } & \multicolumn{3}{|c|}{ Points } & \multirow[t]{2}{*}{ Total } \\
\hline & $\mathbf{0}$ & 1 & 2 & \\
\hline Heart rate, beats per min & $<90$ & $90-109$ & $\geq 110$ & \\
\hline Breathing frequency, breaths/min & $\leq 18$ & $19-30$ & $>30$ & \\
\hline Restlessness $^{\mathrm{a}}$ & None & $\begin{array}{l}\text { Occasional, slight } \\
\text { movements }\end{array}$ & Frequent movements & \\
\hline Accessory muscle use $^{b}$ & None & Slight rise & Pronounced rise & \\
\hline Paradoxical breathing pattern $^{\mathrm{c}}$ & None & NA & Present & \\
\hline Grunting at end-expiration $^{\mathrm{d}}$ & None & NA & Present & \\
\hline Nasal flaring $^{\mathrm{e}}$ & None & NA & Present & \\
\hline Look of fear & None & NA & $\begin{array}{l}\text { Eyes wide open, facial } \\
\text { muscles tense, brow } \\
\text { furrowed, mouth open }\end{array}$ & \\
\hline Total & & & & \\
\hline
\end{tabular}

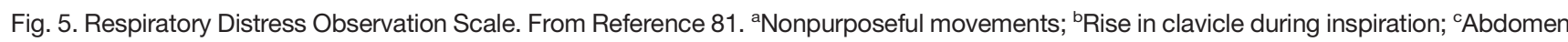
moves in during inspiration; ' ${ }^{\mathrm{G}}$ Guttural sound; Involuntary movement of nares; NA = not applicable.

support levels. The authors concluded that, compared to breathing pattern, $\mathrm{P}_{0.1}$ may be a more sensitive parameter in determining the optimal level of pressure support for patients. Although this is potentially very useful, more research is needed to inform clinicians when to include $\mathrm{P}_{0.1}$ in clinical practice and demonstrate its clinical efficiency and impact on patient outcome.

\section{Monitoring Dyspnea}

Dyspnea is a common symptom manifested by individuals in respiratory distress due to cardiopulmonary and neurologic diseases. It is a strong predictor of mortality and affects $25-50 \%$ patients admitted in ambulatory, acute, and tertiary care units. ${ }^{77}$ Dyspnea is a subjective symptom perceived by patients in respiratory distress, whereas tachypnea, accessory muscle use, diaphoresis, etc., are signs caused by difficulty breathing. The presence and intensity of dyspnea are commonly assessed with visual analog scales, but the assessment of dyspnea is challenging due to its subjective nature.

Similar to pain, dyspnea causes physical suffering and emotional distress to patients. Its identification is crucial for symptom management. Although dyspnea is not uncommon, published literature on the topic suggests that clinicians often underestimate its presence. ${ }^{78-80}$ Binks et $\mathrm{al}^{78}$ reported that significant breathing discomfort is as common as pain in mechanically ventilated subjects and that clinicians misjudge its occurrence. In a prospective, multi-center study, Haugdahl and colleagues ${ }^{79}$ assessed agreement between physicians, nurses, and mechanically ventilated subjects' rated breathlessness during spontaneous breathing trials. The results indicated that subjects reported more breathlessness after undergoing spontaneous breathing trials compared to assessments made by physicians and nurses. More recently, Gentzler et $\mathrm{al}^{80}$ assessed the frequency of dyspnea relative to pain as well as the accuracy of nurses' and personal caregivers' dyspnea ratings relative to patient-reported dyspnea in subjects admitted to the medical ICU. The study results indicated that $47 \%$ of subjects reported moderate-to-severe dyspnea and $41 \%$ reported moderate to severe pain. Furthermore, the authors reported that a subjects' dyspnea rating had significant agreement with a personal caregiver's rating, whereas dyspnea ratings by nurses were not significantly related to subjects' rating. Also, pain was more likely to be treated than was dyspnea.

There is a clinical need to develop and utilize measurement tools that could be utilized to accurately quantify dyspnea. The Respiratory Distress Observation Scale (Fig. 5) is a 8-item, valid, and reliable scale that is used to measure dyspnea in patients who are unable to self-report. ${ }^{81}$ Persichini et al ${ }^{82}$ reported a significant correlation between the visual analog scale, commonly used to assess dyspnea, and the Respiratory Distress Observation Scale to measure dyspnea in subjects admitted in the ICU. This study provides evidence that dyspnea can be assessed quantitatively and accurately. Timely identification of dyspnea could lead to appropriate symptom management and reduce patient discomfort.

For mechanically ventilated patients, questioning their dyspnea level is both doable and useful. In 2011, Schmidt et $\mathrm{al}^{83}$ noted that dyspnea during mechanical ventilation is common and intense and that it was associated with anxiety and mechanical ventilation settings. Perhaps most importantly, it was associated with delayed extubation when dyspnea did not improve with adjustments in ventilator settings. Currently, there is a need for further largescale studies to quantify dyspnea and to develop strategies to alleviate the sensation of breathlessness and improve patient outcomes related to dyspnea, such as quality of life.

\section{Conclusions}

Respiratory monitoring provides clinicians with vital information that aids in clinical decision making. Although they are clinically very important, monitoring parameters 
such as $f$, minute volume, breathing effort, and dyspnea pose significant clinical challenges. Clinicians need to overcome these challenges through education or reeducation, improvements in workflow, and technological advances. Future studies are needed to inform clinicians on better, more practical ways to assess these important clinical values.

\section{REFERENCES}

1. McCarthy M, Shelledy DC. Physical assessment. In: Shelledy DC, Peters JL, eds. Respiratory care: patient assessment and care plan development. Burlington, MA: Jones and Bartlett Learning; 2016:137186.

2. Parkes R. Rate of respiration: the forgotten vital sign. Emerg Nurse 2011;19(2):1-7.

3. Cretikos MA, Bellomo R, Hillman K, Chen J, Finfer S, Flabouris A. Respiratory rate: the neglected vital sign. Med J Aust 2008;188 (11):657-659.

4. Kellett J, Sebat F. Make vital signs great again: a call for action. Eur J Intern Med 2017;45:13-19.

5. Fieselmann JF, Hendryx MS, Helms CM, Wakefield DS. Respiratory rate predicts cardiopulmonary arrest for internal medicine patients. J Gen Intern Med 1993;8(7):354-360.

6. Subbe CP, Davies RG, Williams E, Rutherford P, Gemmell L. Effect of introducing the Modified Early Warning score on clinical outcomes, cardio-pulmonary arrests and intensive care utilisation in acute medical admissions. Anaesthesia 2003;58(8):797-802.

7. Goldhill DR, McNarry AF, Mandersloot G, McGinley A. A physiologically-based early warning score for ward patients: the association between score and outcome. Anaesthesia 2005;60(6):547-553.

8. Ansell H, Meyer A, Thompson S. Why don't nurses consistently take patient respiratory rates? Br J Nurs 2014;23(8):414-418.

9. Flenady T, Dwyer T, Applegarth J. Explaining transgression in respiratory rate observation methods in the emergency department: a classic grounded theory analysis. Int J Nurs Stud 2017;74:67-75.

10. Gravel J, Opatrny L, Gouin S. High rate of missing vital signs data at triage in a paediatric emergency department. Paediatr Child Health 2006;11(4):211-215.

11. Lovett PB, Buchwald JM, Stürmann K, Bijur P. The vexatious vital: neither clinical measurements by nurses nor an electronic monitor provides accurate measurements of respiratory rate in triage. Ann Emerg Med 2005;45(1):68-76.

12. Mukkamala SG, Gennings C, Wenzel RP. $\mathrm{R}=20$ : bias in the reporting of respiratory rates. Am J Emerg Med 2008;26(2):237-239.

13. Semler MW, Stover DG, Copland AP, Hong G, Johnson MJ, Kriss MS, et al. Flash mob research: a single-day, multicenter, residentdirected study of respiratory rate. Chest 2013;143(6):1740-1744.

14. Philip K, Pack E, Cambiano V, Rollmann H, Weil S, O’Beirne J. The accuracy of respiratory rate assessment by doctors in a London teaching hospital: a cross-sectional study. J Clin Monit Comput 2015;29 (4):455-460.

15. Rolfe S. The importance of respiratory rate monitoring. Br J Nurs 2019;28(8):504-508.

16. Flenady T, Dwyer T, Applegarth J. Accurate respiratory rates count: so should you. Australas Emerg Nurs J 2017;20(1):45-47.

17. Cardona-Morrell M, Prgomet M, Lake R, Nicholson M, Harrison R, Long $\mathrm{J}$, et al. Vital signs monitoring and nurse-patient interaction: a qualitative observational study of hospital practice. Int J Nurs Stud 2016;56:9-16.

18. Philip K, Richardson R, Cohen M. Staff perceptions of respiratory rate measurement in a general hospital. Br J Nurs 2013;22(10):570-574.
19. Takayama A, Takeshima T, Nakashima Y, Yoshidomi T, Nagamine T, Kotani K. A comparison of methods to count breathing frequency. Respir Care 2019;64(5):555-563.

20. Siobal MS. Monitoring exhaled carbon dioxide. Respir Care 2016;61 (10):1397-1416.

21. Carlisle H. Promoting the use of capnography in acute care settings: an evidence-based practice project. J Perianesth Nurs 2015;30(3):201208.

22. Waugh JB, Epps CA, Khodneva YA. Capnography enhances surveillance of respiratory events during procedural sedation: a meta-analysis. J Clin Anesth 2011;23(3):189-196.

23. Overdyk F, Guerra JJ. Improving outcomes in med-surg patients with opioid induced respiratory depression. Am Nurse Today 2011;6: 26-30.

24. Jarzyna D, Jungquist CR, Pasero C, Willens JS, Nisbet A, Oakes L, et al. American Society for Pain Management Nursing guidelines on monitoring for opioid-induced sedation and respiratory depression. Pain Manag Nurs 2011;12(3):118-145.

25. Gottlieb M, Rice M. What is the utility of end-tidal capnography for procedural sedation and analgesia in the emergency department? Ann Emerg Med 2017;70(6):819-821.

26. Wall BF, Magee K, Campbell SG, Zed PJ. Capnography versus standard monitoring for emergency department procedural sedation and analgesia. Cochrane Database Syst Rev 2017;3(3):CD010698.

27. Campbell SG, Magee KD, Zed PJ, Froese P, Etsell G, LaPierre A, et al. End-tidal capnometry during emergency department procedural sedation and analgesia: a randomized, controlled study. World J Emerg Med 2016;7(1):13-18.

28. Gallagher JJ. Capnography monitoring during procedural sedation and analgesia. AACN Adv Crit Care 2018;29(4):405-414.

29. Mandel JE. Recent advances in respiratory monitory in nonoperating room anesthesia. Curr Opin Anesthesiol 2018;31:448-452.

30. Mimoz O, Benard T, Gaucher A, Frasca D, Debaene B. Accuracy of respiratory rate monitoring using a non-invasive acoustic method after general anaesthesia. Br J Anaesth 2012;108(5):872-875.

31. Suzuki T, Tsuda S, Nakae H, Imai J, Sawamoto K, Kijima M, et al. Usefulness of acoustic monitoring of respiratory rate in patients undergoing endoscopic submucosal dissection. Gastroenterol Res Pract 2016;2016:2964581.

32. Tanaka P, Tanaka M, Drover D. Detection of respiratory compromise by acoustic monitoring, capnography, and brain function monitoring during monitored anesthesia care. J Clin Monit Comput 2014;28(6): 561-566.

33. Ramsay MA, Usman M, Lagow E, Mendoza M, Untalan E, De Vol E. The accuracy, precision and reliability of measuring ventilatory rate and detecting ventilatory pause by rainbow acoustic monitoring and capnometry. Anesth Analg 2013;117(1):69-75.

34. Ouchi K, Fujiwara S, Sugiyama K. Acoustic method respiratory rate monitoring is useful in patients under intravenous anesthesia. J Clin Monit Comput 2017;31(1):59-65.

35. Yang S, Menne A, Hu P, Stansbury L, Gao C, Dorsey N, et al. Acoustic sensor versus electrocardiographically derived respiratory rate in unstable trauma patients. J Clin Monit Comput 2017;31(4):765-772.

36. Ishikawa M, Namizato D, Sakamoto A. The value of acoustic respiratory rate monitoring in a patient with postoperative hemorrhage after thyroidectomy: a case report. J Clin Monit Comput 2020;34(1):147150 .

37. McGrath SP, Pyke J, Taenzer AH. Assessment of continuous acoustic respiratory rate monitoring as an addition to a pulse oximetry-based patient surveillance system. J Clin Monit Comput 2017;31(3):561-569.

38. Eisenberg ME, Givony D, Levin R. Acoustic respiration rate and pulse oximetry-derived respiration rate: a clinical comparison study. J Clin Monit Comput 2020;34(1):139-146. 
39. Guechi Y, Pichot A, Frasca D, Rayeh-Pelardy F, Lardeur J, Mimoz O. Assessment of noninvasive acoustic respiration rate monitoring in patients admitted to an emergency department for drug or alcoholic poisoning. J Clin Monit Comput 2015;29(6):721-726.

40. Frasca D, Geraud L, Charriere JM, Debaene B, Mimoz O. Comparison of acoustic and impedance methods with mask capnometry to assess respiration rate in obese patients recovering from general anaesthesia. Anaesthesia 2015;70(1):26-31.

41. Subbe CP, Kinsella S. Continuous monitoring of respiratory rate in emergency admissions: evaluation of the RespiraSense ${ }^{\mathrm{TM}}$ sensor in acute care compared to the industry standard and gold standard. Sensors 2018;18(8):2700.

42. Braun SR. Respiratory rate and pattern. In: Walker HK, Hall WD, Hurst JW, eds. Clinical methods: the history, physical, and laboratory examinations, third edition. Boston: Butterworth Publishers; 1990.

43. Lynn LA, Curry JP. Patterns of unexpected in-hospital deaths: a root cause analysis. Patient Saf Surg 2011;5(1):3.

44. Gilbert R, Auchincloss JH Jr, Brodsky J, Boden W. Changes in tidal volume, frequency, and ventilation induced by their measurement. $\mathrm{J}$ Appl Physiol 1972;33(2):252-254.

45. Kodali BS, Choi L, Chau A, Harvey BC, Brayanov J, Tsen LC, et al. Use of a novel non-invasive respiratory monitor to study changes in pulmonary ventilation during labor epidural analgesia. J Clin Monit Comput 2019 [Epub ahead of print].

46. Holley K, MacNabb CM, Georgiadis P, Minasyan H, Shukla A, Mathews $\mathrm{D}$. Monitoring minute ventilation versus respiratory rate to measure the adequacy of ventilation in patients undergoing upper endoscopic procedures. J Clin Monit Comput 2016;30(1):33-39.

47. Cavalcante AN, Martin YN, Sprung J, Imsirovic J, Weingarten TN. Low minute ventilation episodes during anesthesia recovery following intraperitoneal surgery as detected by a non-invasive respiratory volume monitor. J Clin Monit Comput 2018;32(5):929-935.

48. Schlesinger JJ. Applications of a noninvasive respiratory volume monitor for critical care medicine. Respir Care 2015;60(5):e97-e100.

49. Voscopoulos C, Brayanov J, Ladd D, Lalli M, Panasyuk A, Freeman J. Special article: evaluation of a novel noninvasive respiration monitor providing continuous measurement of minute ventilation in ambulatory subjects in a variety of clinical scenarios. Anesth Analg 2013; 117(1):91-100.

50. Voscopoulos CJ, MacNabb CM, Brayanov J, Qin L, Freeman J, Mullen GJ, et al. The evaluation of a non-invasive respiratory volume monitor in surgical patients undergoing elective surgery with general anesthesia. J Clin Monit Comput 2015;29(2):223-230.

51. Nichols RH, Blinn JA, Ho TM, McQuitty RA, Kinsky MP. Respiratory volume monitoring reduces hypoventilation and apnea in subjects undergoing procedural sedation. Respir Care 2018;63(4):448-454.

52. Mehta JH, Cattano D, Brayanov JB, George EE. Assessment of perioperative minute ventilation in obese versus non-obese patients with a noninvasive respiratory volume monitor. BMC Anesthesiol 2017;17(1):61.

53. Ianchulev S, Ladd D, MacNabb CM, Qin L, Marengi N, Freeman J. Use of a respiratory volume monitor to assess respiratory competence in cardiac surgery patients after extubation. J Clin Med Res 2017;9 (1): 17-22.

54. Kaur R, Vines DL, Liu L, Balk RA. Role of Integrated Pulmonary Index in identifying extubation failure. Respir Care 2017;62(12):15501556.

55. de Vries H, Jonkman A, Shi ZH, Spoelstra-de Man A, Heunks L. Assessing breathing effort in mechanical ventilation: physiology and clinical implications. Ann Transl Med 2018;6(19):387.

56. Aldrich TK. Respiratory muscle fatigue. Clin Chest Med 1988;9(2): 225-236.

57. Marini JJ. Monitoring breathing effort: a work in progress. Crit Care Med 2013;41(6):1584-1586.
58. Cohen CA, Zagelbaum G, Gross D, Roussos C, Macklem PT. Clinical manifestations of inspiratory muscle fatigue. Am J Med 1982;73 (3):308-316.

59. Tulaimat A, Patel A, Wisniewski M, Gueret R. The validity and reliability of the clinical assessment of increased work of breathing in acutely ill patients. J Crit Care 2016;34:111-115.

60. Sklar MC, Burns K, Rittayamai N, Lanys A, Rauseo M, Chen L, et al. Effort to breathe with various spontaneous breathing trial techniques: a physiologic meta-analysis. Am J Respir Crit Care Med 2017;195 (11):1477-1485.

61. Benditt JO. Esophageal and gastric pressure measurements. Respir Care 2005;50(1):68-75.

62. Grinnan DC, Truwit JD. Clinical review: respiratory mechanics in spontaneous and assisted ventilation. Crit Care 2005;9(5):472-484.

63. Brochard L, Rua F, Lorino H, Lemaire F, Harf A. Inspiratory pressure support compensates for the additional work of breathing caused by the endotracheal tube. Anesthesiology 1991;75(5):739-745.

64. Deye N, Lellouche F, Maggiore SM, Taille S, Demoule A, L'Her E, et al. The semi-seated position slightly reduces the effort to breathe during difficult weaning. Intensive Care Med 2013;39(1):85-92.

65. Lin MC, Huang CC, Yang CT, Tsai YH, Tsao TC. Pulmonary mechanics in patients with prolonged mechanical ventilation requiring tracheostomy. Anaesth Intensive Care 1999;27(6):581-585.

66. Rodvold DM, McLeod DG, Brandt JM, Snow PB, Murphy GP. Introduction to artificial neural networks for physicians: taking the lid off the black box. Prostate 2001;46(1):39-44.

67. Banner MJ, Euliano NR, Brennan V, Peters C, Layon AJ, Gabrielli A. Power of breathing determined noninvasively with use of an artificial neural network in patients with respiratory failure. Crit Care Med 2006;34(4):1052-1059.

68. Banner MJ, Euliano NR, Martin AD, Al-Rawas N, Layon AJ, Gabrielli A. Noninvasive work of breathing improves prediction of post-extubation outcome. Intensive Care Med 2012;38(2):248-255.

69. Vivier E, Mekontso Dessap A, Dimassi S, Vargas F, Lyazidi A, Thille AW, et al. Diaphragm ultrasonography to estimate the work of breathing during non-invasive ventilation. Intensive Care Med 2012;38 (5):796-803.

70. Whitelaw WA, Derenne JP, Milic-Emili J. Occlusion pressure as a measure of respiratory center output in conscious man. Respir Physiol 1975;23(2):181-199.

71. Eldridge FL. Relationship between respiratory nerve and muscle activity and muscle force output. J Appl Physiol 1975;39(4):567-574.

72. Whitelaw WA, Derenne JP. Airway occlusion pressure. J Appl Physiol (1985) 1993;74(4):1475-1483.

73. Conti G, Antonelli M, Arzano S, Gasparetto A. Measurement of occlusion pressures in critically ill patients. Crit Care 1997;1(3):89-93.

74. Beloncle F, Piquilloud L, Olivier P-Y, Vuillermoz A, Yvin E, Mercat A, et al. Accuracy of P0.1 measurements performed by ICU ventilators: a bench study. Ann Intensive Care 2019;9(1):104.

75. Laghi F. Assessment of respiratory output in mechanically ventilated patients. Respir Care Clin N Am 2005;11(2):173-199.

76. Alberti A, Gallo F, Fongaro A, Valenti S, Rossi A. P0.1 is a useful parameter in setting the level of pressure support ventilation. Intensive Care Med 1995;21(7):547-553.

77. Parshall MB, Schwartzstein RM, Adams L, Banzett RB, Manning HL, Bourbeau J, et al. An official American Thoracic Society statement: update on the mechanisms, assessment, and management of dyspnea. Am J Respir Crit Care Med 2012;185(4):435-452.

78. Binks AP, Desjardin S, Riker R. ICU clinicians underestimate breathing discomfort in ventilated subjects. Respir Care 2017;62 (2):150-155.

79. Haugdahl HS, Storli SL, Meland B, Dybwik K, Romild U, Klepstad P Underestimation of patient breathlessness by nurses and physicians 
during a spontaneous breathing trial. Am J Respir Crit Care Med 2015;192(12):1440-1448.

80. Gentzler ER, Derry H, Ouyang DJ, Lief L, Berlin DA, Xu CJ, et al. Underdetection and undertreatment of dyspnea in critically ill patients. Am J Respir Crit Care Med 2019;199(11):1377-1384.

81. Campbell ML. Psychometric testing of a respiratory distress observation scale. J Palliat Med 2008;11(1):44-50.
82. Persichini R, Gay F, Schmidt M, Mayaux J, Demoule A, MorelotPanzini C, et al. Diagnostic accuracy of respiratory distress observation scales as surrogates of dyspnea self-report in intensive care unit patients. Anesthesiology 2015;123(4):830-837.

83. Schmidt M, Demoule A, Polito A, Porchet R, Aboab J, Siami S, et al. Dyspnea in mechanically ventilated critically ill patients. Crit Care Med 2011;39(9):2059-2065.

\section{Discussion}

Schmidt: I wanted to raise the idea that, while it seems self-evident, that a more accurate breathing frequency is better. There's an interesting study that suggests there's an extra element we ought to consider especially if the question is, 'is frequency a good predictor of trouble to come?"1 which is what Fieselmann's paper ${ }^{2}$ from many years ago is about. It comes from a paper that looked at an electronic means of measuring frequency and heart rate and compared that to the documentation of the nurses in the electronic health record. ${ }^{1}$ One of the findings was that the median frequency documented by the nurses was 18 breaths/min. It was a very large study, by the way, of more than 1,000 subjects. But it turned out that what the nurses documented was a better predictor of deterioration than the presumably more accurate electronic measure, and they hypothesized that there was some other element going on; that it wasn't just frequency and that when nurses write a higher frequency down there's something else other than frequency that they recognized that caused them to document the frequency. Maybe it's just that nurses need to be defended, but I think there's some extra element in the nurses' documented frequency compared to truth.

Scott: Very interesting, and this is certainly not an effort to put to focus solely on nurses. A lot of the papers presented here were from Australia/New Zealand and some European countries where respiratory therapists do not practice. But, to your point, it sounds like maybe there's nursing intuition factor that may need to be accounted for here.
Smallwood: These data are very interesting. I have a couple questions though. I agree that there is something to be said for nursing or clinician intuition. Perhaps this is why we don't see a ton of automated systems outperforming bedside clinicians. But what if it's something else? Given how we collect information at the bedside and the issues with documenting erroneous breathing frequency values as has been presented, how could a system possibly detect a deterioration in patient condition earlier if it's only able to look at documented data? Data that actually doesn't reflect what's happening with the patient. If I do a study that relies upon this manually transcribed data, data that is only entered into the record when the bedside clinician deems it necessary and is possibly or even likely to be erroneous, why should I be surprised that I am unable to build something that does better than the clinician? That's the question I'm asking. I don't know exactly where to go with that, but we've all heard the expression before 'garbage in, garbage out'. That brings up the point, are there data that a clinician sees that aren't in the record? For sure. But where do we go from here? This is an open question that has me thinking.

Rackley: It's important because breathing frequency and altered mental status are two of the biggest predictors of decompensation and early sepsis, and make up two-thirds of the qSOFA score. ${ }^{3}$ Clinical prediction scores, such as the qSOFA and NEWS scores ${ }^{4}$ are becoming part of the medical record and function as an early warning that the patient is about to get sick. I wonder if the nurse is recognizing a slight altered mentation and that is causing them to document breathing frequency a bit more accurately. However, it is interesting that you mentioned agitation and altered mental status as reasons given for NOT recording the breathing frequency. I also think not recognizing the patient's dyspnea can be an important oversight. When you assess patients who required prolonged mechanical ventilation several months after recovery, a third of them remember being short of breath and having difficulty communicating. ${ }^{5}$ We also know that family members are often better at assessing a patient's dyspnea than providers, so appreciating this is probably important. ${ }^{6}$ I want to ask you a question; What do you commonly see dyspnea interpreted as by the nurses or house staff?

Scott: I've had a lot of nurses just simply ask, "Can you come look at my patient?, Something doesn't seem right." But that seems to be associated with visual signs of shortness of breath, mental status change, or confusion. Which is probably what you see, too.

Rackley: I commonly see dyspnea, tachypnea, and early sepsis interpreted as anxiety. Dr MacIntyre and I were in the intensive care unit the other day, and a patient had a $\mathrm{pH}$ of 7.28. The staff reported, 'oh they're not just short of breath, they're really anxious'. A pH of 7.28 will make a patient very short of breath and I think misinterpreting this as anxiety instead of dyspnea can lead to treatment of anxiety rather than recognizing an early sign of sepsis or impending decompensation. 
Scott: The more I thought about this while going through the literature, especially the qualitative literature, it really is hard to stand there and count frequency for 1 minute. It's just not easy to capture, for various reasons. This presentation and literature search is making me wonder if while placing blood pressure cuffs and EKG leads, we should be applying some type of device to accurately quantify and track breathing frequency. I understand that patient assessment is the key to this, and I'm all about it a good clinical assessment, but clearly there's an issue with accuracy and documentation.

*Hess: There is a pulse oximeter that measures breathing frequency by looking at the artifacts that Greg [Schmidt] talked about this morning. Nobody would omit getting a pulse oximeter saturation, so why not get the breathing frequency off the same device?

Scott: Agreed. That was one of the devices that I presented earlier, and it seems to work pretty well. It's interesting technology.

Pham: Regarding breathing frequency, I feel like you have lots of variability with patients, which is normal and not concerning. Maybe repeating the same measurements with different devices at different times is actually not accurate. The several assessments might not actually be different but just a variability of the patient. Second, if you want to be sure that your patient will not desaturate or have no signs of dyspnea and then respiratory failure because they have a high breathing frequency, I feel like having a frequency even if it's eyeballing and seeing that the patient doesn't seem like they're tachypneic would be ok. There are different settings where you may want to closely observe oxygenation because you're applying high-flow or for research purposes you need a more accurate measurements, but otherwise
I would propose that you might not want such a precise frequency.

Schmidt: There isn't just frequency either. Every breath cycle is potentially different and just as you alluded to, the variability may be an important signal about stress on the system just like heart rate variability. And if we think of frequency as 18 breaths/min vs 27 breaths/min that's one thing, but you might think about two similar frequencies being different if the amount of variability is different. Although I argued a few moments ago against accurate measurements because the nurse adds value, there might be something about variability that would add to just the number of breaths/min.

Walsh: One of the things I worry about is we have a hard time determining work of breathing. It's not just a frequency of 18 breaths/min. Maybe that's what the nurses are picking up on that is predicting the downward spiral. Did you see anything about the pattern of breathing or other estimations of work of breathing? Maybe taken from EKG monitoring, like pattern recognition (slope), I know some people have been looking at that over the years. I do believe that if you have a pretty nice breathing pattern, just like pulse oximetry with a nice plethysmograph, then you can believe the pulse oximeter number. So I don't know why we don't apply that to frequency, if we can see that there's a decent pattern and the patient is still, then you can believe that number versus doing a whole minute calculation to get that number. Or allowing an early warning system to use.

Scott: One thing that happens with those monitors is that occasionally the breathing frequency alarms will activate and display something erroneous like, apnea or zero, even though the patient is intubated and mechanically ventilated. So, I can tell you that at this point in time, I have very little confidence in the EKG and its ability to accurately display breathing frequency. I've personally seen patients on mechanical ventilation and the breathing frequency displayed on the physiologic monitor was totally inaccurate. So, for this presentation, I did not include it.

$\dagger$ Kallstrom: Did you look at the inability to say a complete sentence, maybe a three word sentence? Is there much in the literature about that when it comes to determining dyspnea?

Scott: I think when you look at the dyspnea papers presented here, speaking in short sentences was definitely a component. What I gained from the literature was that dyspnea is a bit challenging to determine. Clinically, we certainly acknowledge that if somebody can't speak in full sentences prior to treatments, and then they can afterwards, it certainly signals and improvement in their degree of dyspnea.

Walsh: In support of our nursing colleagues, I remember a study about RTs documenting heart rate when they were doing treatments and they would chart the breathing frequency almost $100 \%$ of the time but not document the heart rate. So you wonder if the nurses were doing the same thing because that's their focus. If it's in the normal range maybe they drop some of the other items just like RTs do.

Scott: Again, my intent is not to target our nursing colleagues. It's just the group most often involved in the literature we have on the topic. I suspect that if you did the same studies with RTs, you would find similar results.

\section{REFERENCES}

1. Churpek MM, Snyder A, Twu NM, Edelson DP: Accuracy comparisons between manual and automated respiratory rate for detecting 
clinical deterioration in ward patients. J Hosp Med 2018; 13(7): 486-487.

2. Fieselmann JF, Hendryx MS, Helms CM, Wakefield DS: Respiratory rate predicts cardiopulmonary arrest for internal medicine patients. J Gen Intern Med 1993; 8(7): 354-360.

3. Seymour CW, Liu VX, Iwashyna TJ, Brunkhorst FM, Rea TD, Scherag A, et al. Assessment of clinical criteria for sepsis: For the Third International Consensus Definitions for Sepsis and Septic Shock (Sepsis-3). JAMA 2016;315(8):762-774.
4. Prytherch DR, Smith GB, Schmidt PE, Featherstone PI. ViEWS-Towards a national early warning score for detecting adult inpatient deterioration. Resuscitation 2010;81 (8):932-937.

5. Jubran A, Grant BJB, Duffner LA, Collins EG, Lanuza DM, Hoffman LA, Tobin MJ. Long-term outcome after prolonged mechanical ventilation. a long-term acutecare hospital study. Am J Respir Crit Care Med 2019;199(12):

1508-1516.
6. Gentzler ER, Derry H, Ouyang DJ, Lief L, Berlin DA, Xu CJ, Maciejewski PK, Prigerson HG. Underdetection and undertreatment of dyspnea in critically ill patients. Am J Respir Crit Care Med 2019;199 (11):1377-1384.

*Dean R Hess PhD RRT FAARC is Managing Editor of RESPIRATORY CARE.

$\uparrow$ Thomas J Kallstrom MBA RRT FAARC is Executive Director of the AARC and Publisher of RESPIRATORY CARE.

This article is approved for Continuing Respiratory Care Education credit. For information and to obtain your CRCE

(free to AARC members) visit 